
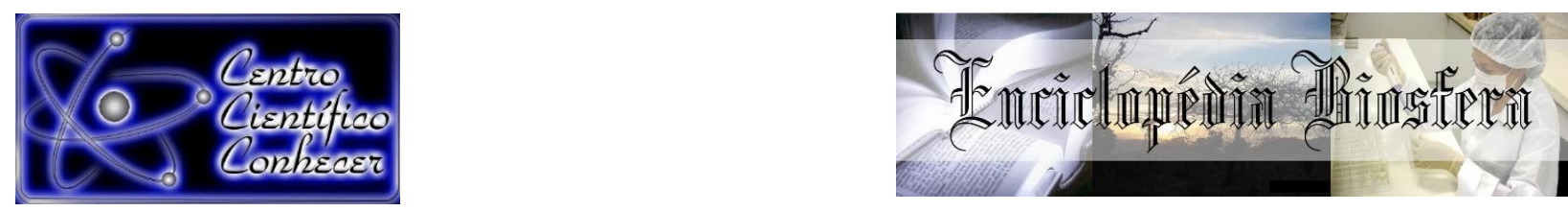

\title{
CONSUMO DE PROCESSADOS E ULTRAPROCESSADOS POR GESTANTES DE UM MUNICÍPIO DO TOCANTINS
}

\author{
Larissa Suriany de Almeida Campos ${ }^{1}$, Mariana Ferreira e Castro ${ }^{1}$, Brunna Tayná \\ Soares Pinheiro ${ }^{1}$, Fabiana Cândida Queiroz Santos Anjos ${ }^{2}$, Renata Junqueira \\ Pereira $^{3}$ \\ 1 Nutricionista pela Universidade Federal do Tocantins \\ 2 Mestre em Ciências da Saúde pela Universidade Federal do Tocantins \\ 3 Doutora em Ciência dos Alimentos e docente do curso de Nutrição da \\ Universidade Federal do Tocantins. Autora para correspondência. E-mail: \\ renatajunqueira@uft.edu.br
}

Recebido em: 15/02/2020 - Aprovado em: 15/03/2020 - Publicado em: 30/03/2020 DOI: 10.18677/EnciBio_2020A5

\begin{abstract}
RESUMO
O consumo alimentar na gestação é relevante e determinante para o estado nutricional, tanto materno, quanto do feto podendo impactar em seu desenvolvimento. Diante disso, o presente estudo avaliou o consumo alimentar de 46 gestantes, por meio da aplicação de um questionário de frequência de consumo alimentar (QFCA) e um questionário sobre a gestação atual, com o objetivo de avaliar o consumo de alimentos processados e ultraprocessados e sua influência sobre o estado nutricional materno. Os dados foram tabulados no Microsoft Excel e, posteriormente, analisados no Statistical Package of Social Sciences, versão 20.0, utilizando-se o teste do Qui-Quadrado de Pearson, a 5\% de significância. Observouse associação significativa entre o consumo de lipídeos, proteínas e energia e alterações do estado nutricional materno. Quanto maior o percentual de lipídeos e proteínas oriundos de alimentos processados e ultraprocessados na dieta, pior o estado nutricional materno. O consumo de macronutrientes advindos desses tipos de alimentos correlacionaram-se, em sua maioria, de forma negativa ao estado nutricional materno, sendo o ganho de peso e o índice de massa corporal afetados significativamente.
\end{abstract}

PALAVRAS-CHAVE: Alimentos processados. Estado Nucicional. Gestação

\section{CONSUMPTION OF PROCESSED FOOD AND ASSOCIATED FACTORS IN PREGNANTS OF TOCANTINS}

\begin{abstract}
Food intake in pregnancy is relevant and determinant for both maternal and fetal nutritional status and may impact its development. Therefore, the present study evaluated the food intake of 46 pregnant women, by applying a food consumption frequency questionnaire (FCFQ) and a questionnaire about the current pregnancy, with the objective of evaluating the consumption of processed and ultra-processed foods. its influence on maternal nutritional status. Data were tabulated in Microsoft Excel and later analyzed in the Statistical Package of Social Sciences, version 20.0,
\end{abstract}


using Pearson's chi-square test at 5\% significance. Significant association was observed between lipid, protein and energy consumption and changes in maternal nutritional status. The higher the percentage of lipids and proteins from processed and ultra-processed foods in the diet, the worse the maternal nutritional status. The consumption of macronutrients from these types of food correlated mostly negatively with maternal nutritional status, with weight gain and body mass index significantly affected.

KEYWORDS: Gestation. Nutritional status. Processed food.

\section{INTRODUÇÃO}

A gestação é um período marcado por inúmeras alterações fisiológicas, metabólicas e nutricionais no corpo da mulher, preparando-o para o desenvolvimento e crescimento do feto. Nesse momento, as necessidades energéticas aumentam consideravelmente, incluindo carboidratos, proteínas e lipídeos, além de alguns micronutrientes essenciais no período gravídico. Além disso, a qualidade da alimentação da gestante e seu consequente estado nutricional exercem grande relevância no crescimento do feto e nas condições de peso ao nascer (CUNHA et al., 2016).

O estado nutricional materno, assim como o ganho de peso gestacional, tem ganhado grande enfoque nos estudos e pesquisas atuais, não somente pela crescente prevalência dos distúrbios, mas, sobretudo devido ao papel determinante sobre os desfechos gestacionais (CAMPOS et al., 2019). Uma das principais adversidades que acometem a gestação é a obesidade materna e o ganho de peso acima do recomendado, fatores esses que aumentam substancialmente os riscos de diabetes gestacional (DM), parto prolongado, pré-eclâmpsia, cesárea e depressão (CAMPOS et al., 2019).

Por outro lado, o baixo peso materno, associado ao insuficiente ganho de peso durante a gestação também influenciam negativamente no peso e nas condições de saúde da criança ao nascer, constituindo-se em fatores de risco modificáveis gestacionais mais importantes. Nesse sentido, observa-se uma significativa influência do comportamento alimentar materno nas condições nutricionais de seu corpo e, consequentemente, nos desfechos da gestação (CUNHA et al., 2016).

Em 2016 foi publicada a NOVA, uma classificação alimentar que classificou os produtos alimentícios e alimentos, não só levando em consideração os teores de nutrientes, mas também o tipo de processamento empregado até o produto final. Os quatro grupos classificados pela NOVA são alimentos in natura ou minimamente processados; ingredientes culinários processados, alimentos processados e alimentos ultraprocessados (MONTEIRO et al., 2016).

São considerados alimentos in natura e minimamente processados quaisquer partes comestíveis de animais e plantas, logo após a retirada da natureza. Os alimentos que passam por processos de lavagem, descascamentos, pasteurização, fracionamento e desidratação também fazem parte desse grupo. Os ingredientes culinários processados, são aqueles ingeridos como ingredientes de preparações culinárias; tais substâncias passam por processos como moagem, secagem, refino, prensagem ou pulverização para que sejam extraídas, em geral de alimentos presentes no grupo dos in natura ou minimamente processados (MONTEIRO et al., 2016).

Os outros dois grupos são os dos alimentos processados e ultraprocessados. No primeiro se enquadram produtos alimentícios, que passaram 
por processos de adição de substâncias, seja sal, açúcar, óleo, vinagre ou qualquer outra substância. Já o último grupo, contempla os alimentos ultraprocessados, definidos como aqueles em que já não há quaisquer resquícios dos alimentos in natura, sendo produtos completamente industriais e, em geral, compostos por cinco ou mais ingredientes (MONTEIRO et al., 2016).

Dentre os grupos da classificação NOVA, os alimentos ultraprocessados destacamse como os mais prejudiciais à saúde humana, por possuírem uma maior densidade energética e teores elevados de açúcar e sódio, além de menores teores de fibras, quando comparados aos outros três grupos (LOUZADA et al., 2015). Nesse sentido, o objetivo desse estudo foi avaliar o consumo de alimentos processados e ultraprocessados por gestantes, associando-o ao estado nutricional materno.

\section{MATERIAL E MÉTODOS}

Trata-se de estudo transversal, com gestantes de baixo risco, acompanhadas na atenção básica de saúde do município de Gurupi -TO. O município de Gurupi possui 13 Unidades Básicas de Saúde (UBS) e todas as gestantes que consultaram entre os meses de junho e novembro de 2018 foram convidadas a participar do estudo, sendo abordadas 148 gestantes no total. Destas, 96 concordaram em participar do estudo, porém, após aplicação dos critérios de exclusão e devido a perdas por não realizarem todas as etapas da coleta de dados, 46 participantes compuseram a amostra final.

A idade materna inferior a 18 anos, a gestação gemelar, a presença de doenças ou complicações identificadas no decorrer do estudo, como diabetes, hipertensão, alterações da tireoide, doenças absortivas, cirurgias gástricas prévias, foram os critérios de exclusão.

As gestantes foram entrevistadas na UBS, sendo aplicado incialmente um questionário com variáveis sobre características maternas e da gestação atual (idade gestacional (IG), peso pré-gestacional (PPG), peso atual (PA), estatura e ganho de peso, permitindo avaliação dos Índices de Massa Corporal PréGestacional (IMCPG) e atual para a IG.

O estado nutricional pré-gestacional pelo IMC foi classificado conforme proposto pela OMS e o estado nutricional atual pelo IMC por idade gestacional foi classificado conforme proposto por Atalah et al. (1997). O ganho de peso gestacional foi avaliado conforme proposto pelo Institute of Medicine (RASMUSSEN; YAKTINE, 2009).

Foi aplicado um Questionário de Frequência do Consumo Alimentar (QFCA), semiquantitativo, especificamente criado para este estudo e ainda não validado. $O$ QFCA foi composto por grupos de alimentos de consumo nacional e regional, incluindo alimentos in natura, ingredientes culinários, alimentos processados e ultraprocessados, conforme o Guia Alimentar para a População Brasileira e a classificação NOVA dos alimentos, que os classifica conforme a extensão e o propósito do processamento. Os alimentos foram dispostos de forma a avaliar a frequência do consumo e o tamanho da porção consumida. O QFCA foi aplicado com o auxílio de um manual fotográfico de quantificação alimentar (CRISPIM et al., 2017).

Após transformação e quantificação do consumo alimentar diário, obtido pelo QFCA, e da dupla digitação, foi realizada análise de consistência, verificando-se os alimentos e preparações digitados, com especial atenção às unidades de medida. Em seguida foi utilizado o programa Diet Box para a análise do consumo alimentar de cada uma das participantes, pesquisando-se os alimentos nas tabelas de 
composição química de alimentos disponíveis no software, na seguinte ordem: pesquisou-se o alimento inicialmente na tabela proposta por Philippi (2015) seguida da tabela TACO (NEPA, 2011) e, quando o alimento não foi localizado em nenhuma delas, utilizou-se a tabela do IBGE (BRASIL, 2011).

Para cada paciente foi estimado o consumo diário de energia e macronutrientes total e por grupo de alimentos, conforme a classificação NOVA, que classifica todos os alimentos e produtos alimentícios em quatro grupos, claramente distintos, especificando o tipo de processamento empregado na sua produção e a finalidade: Grupo 1: alimentos in natura ou minimamente processados; Grupo 2: Ingredientes culinários processados; Grupo 3: Alimentos processados; Grupo 4: Alimentos ultraprocessados (MONTEIRO et al., 2016).

A adequação da ingestão total de energia e macronutrientes, conforme a recomendação diária, foi avaliada dentro dos limites recomendados pelas Dietary Reference Intakes (DRI) (COMINETTI; COZZOLINO, 2017). A população foi dividida em tercis para as ingestões absolutas (em gramas por dia) e relativas (em percentuais) de energia e macronutrientes, oriundas dos alimentos processados e ultraprocessados consumidos.

Os dados foram tabulados no Microsoft Excel e posteriormente analisados com o Software Statistical Package of Social Science versão 20.0, sendo expressos em estatísticas descritivas. Os tercis de consumos de energia e macronutrientes, oriundos de processados e ultraprocessados, foram associados às características de estado nutricional maternas (IMC pré-gestacional, estado nutricional, ganho de peso e kcal total) pelo teste do Qui-quadrado de Pearson, ao nível de significância de 5\%. O presente trabalho atendeu aos preceitos éticos estabelecidos na Resolução do Conselho Nacional de Saúde $n^{\circ} 510$ de 2016 e foi aprovado pelo Comitê de Ética e Pesquisa com seres humanos (CEP) da Universidade Federal do Tocantins, parecer 2.600.381. Todas as participantes assinaram o Termo de Consentimento Livre e Esclarecido.

\section{RESULTADOS}

As 46 gestantes que compuseram a amostra apresentaram média de idade $26,65 \pm 5,5$ anos e idade gestacional média de $25,26 \pm 8,6$ semanas, estando a maior parte da amostra no segundo e terceiro trimestres de gestação. O peso prégestacional médio apresentado pelas gestantes foi de $62,89 \pm 13 \mathrm{~kg}$ e o peso atual médio de $67,28 \pm 12,1 \mathrm{~kg}$, conforme pode ser observado na Tabela 1 .

TABELA 1. Características de saúde e estado nutricional de gestantes acompanhadas na atenção básica do município de Gurupi -TO, 2018.

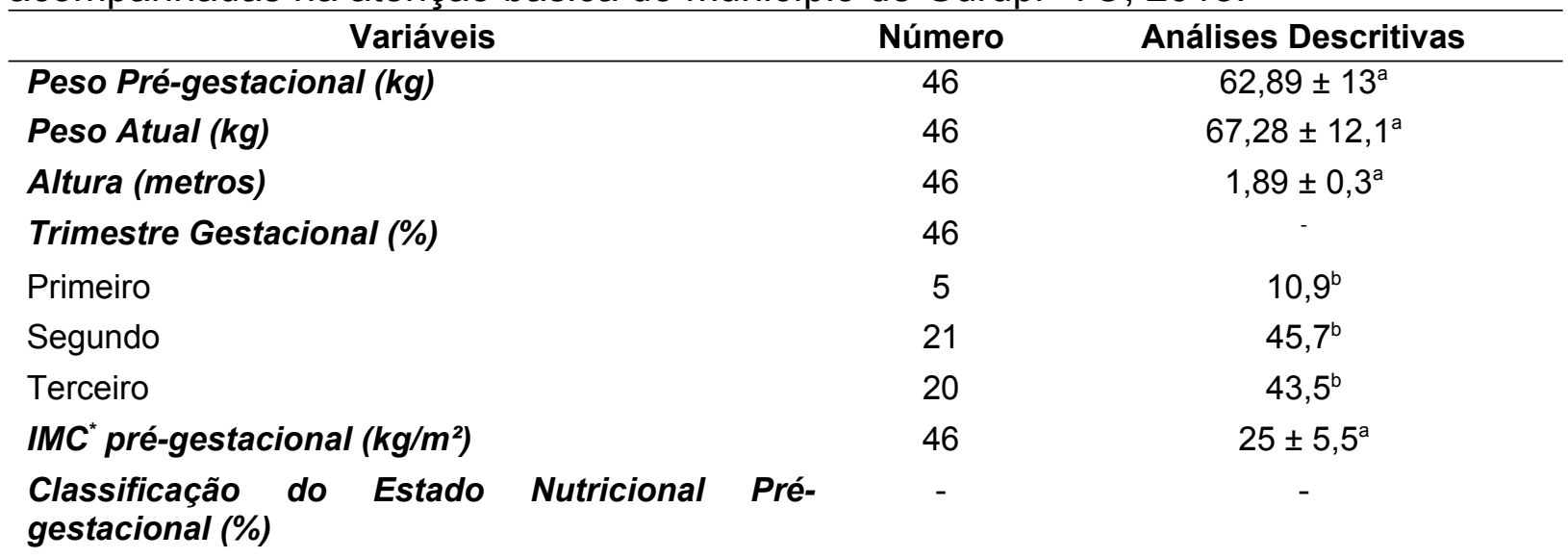




\begin{tabular}{lcc} 
Baixo peso & 4 & $8,7^{\mathrm{b}}$ \\
Eutrofia & 22 & $47,8^{\mathrm{b}}$ \\
Sobrepeso & 13 & $28,3^{\mathrm{b}}$ \\
Obesidade & 7 & $15,2^{\mathrm{b}}$ \\
IMC $^{*}$ por idade gestacional & 46 & $26,74 \pm 5,2^{\mathrm{a}}$ \\
Classificação do Estado Nutricional Atual (\%) & - & - \\
Baixo Peso & 12 & $26,1^{\mathrm{b}}$ \\
Eutrofia & 15 & $32,6^{\mathrm{b}}$ \\
Sobrepeso & 12 & $26,1^{\mathrm{b}}$ \\
Obesidade & 7 & $15,2^{\mathrm{b}}$ \\
Ganho de Peso Gestacional Médio (kg) & 46 & $4,35 \pm 4,9^{\mathrm{a}}$ \\
Classificação do Ganho de Peso Gestacional (\%) & - & - \\
Insuficiente & 25 & $54,3^{\mathrm{b}}$ \\
Adequado & 10 & $21,7^{\mathrm{b}}$ \\
Excessivo & 11 & $23,9^{\mathrm{b}}$ \\
\hline
\end{tabular}

Fonte: Dados da pesquisa.

Nota: ${ }^{a}$ Médias e Desvios-padrão; ${ }^{\text {b }}$ Percentuais.

Notou-se na população estudada, mais de $50 \%$ das gestantes com ganho de peso insuficiente e $78,2 \%$ com ganho de peso inadequado. As prevalências de excesso de peso pré-gestacional e atual totalizaram 43,5\% e 41,3\%, respectivamente. No entanto, o percentual de mulheres com baixo peso aumentou do período pré-gravídico para o atual, sendo que o percentual de gestantes em eutrofia também decresceu, mostrando uma tendência de declínio do estado nutricional das gestantes.

A tabela 2 mostra o perfil de consumo de nutrientes das gestantes estudadas, estimado pelo QFCA semiquantitativo.

TABELA 2. Perfil de consumo de macronutrientes e energia, por 46 gestantes acompanhadas na atenção básica do município de Gurupi -TO, 2018.

\begin{tabular}{|c|c|c|}
\hline Variáveis & $\mathbf{n}$ & Análises Descritivas \\
\hline Consumo médio de Carboidratos (g/dia) & 46 & $390,7 \pm 188^{a}$ \\
\hline $\begin{array}{l}\text { Percentual médio de carboidratos em relação a } \\
\text { energia total ingerida (\%) }\end{array}$ & 46 & $56,3 \pm 8,42^{b}$ \\
\hline Adequação ao AMDR* de carboidratos (\%) & 46 & - \\
\hline Abaixo de $45 \%$ & 6 & $13^{b}$ \\
\hline Entre 45 e $65 \%$ & 34 & $73,9^{b}$ \\
\hline Acima de $65 \%$ & 6 & $13^{b}$ \\
\hline Consumo médio de Lipídeos (g/dia) & 46 & $96,4 \pm 61,7^{a}$ \\
\hline $\begin{array}{l}\text { Percentual médio de lipídeos em relação a energia } \\
\text { total ingerida (\%) }\end{array}$ & 46 & $29,8 \pm 6,8^{b}$ \\
\hline Adequação ao AMDR* de lipídeos (\%) & 46 & - \\
\hline Abaixo de $25 \%$ & 11 & $23,9^{b}$ \\
\hline Entre 25 e $35 \%$ & 24 & $52,2^{\mathrm{b}}$ \\
\hline Acima de $35 \%$ & 11 & $23,9^{b}$ \\
\hline $\begin{array}{l}\text { Consumo médio de proteínas (gramas/kg de peso } \\
\text { corporal/dia) }\end{array}$ & 46 & $1,83 \pm 1,26^{a}$ \\
\hline Consumo médio de Proteínas (g/dia) & 46 & $106,4 \pm 62,3^{a}$ \\
\hline$R D A^{* *}$ média de Proteínas (g/dia) & 46 & $91,4 \pm 15,8^{a}$ \\
\hline$E A R^{\star * *}$ média de Proteínas (g/dia) & 46 & $74 \pm 12,7^{a}$ \\
\hline
\end{tabular}


Adequação à necessidade de proteica (\%)

$<$ EAR

Entre EAR e RDA

$>$ RDA

Energia Média Ingerida (Kcal/dia)

Adequação à necessidade energética estimada

(\%)

$<E E R^{* * * *}$ mínimo

Entre EER mínimo e máximo

$>$ EER máximo $30,4^{b}$

$13^{\mathrm{b}}$

$56,5^{\mathrm{b}}$

$2813 \pm 1422^{a}$

46

46

24

$52,2^{\mathrm{b}}$

18

$39,1^{\text {b }}$

4

$8,7^{\mathrm{b}}$

Fonte: Dados da pesquisa.

Nota: a Médias e Desvios-padrão; ' Percentuais; "AMDR: Acceptable Macronutrient Distribution Ranges - Intervalos aceitáveis de distribuição percentual das calorias consumidas entre os macronutrientes da dieta; ${ }^{* *}$ RDA: Recommended Dietary Allowances - Ingestão Dietética Recomendada para nutrientes; ${ }^{* * *}$ EAR: Estimated Average Requirement - Necessidade Média Estimada para nutrientes ${ }^{* * *}$ EER: Estimated Energy Requirement - Necessidade Energética Estimada.

Observou-se que a maioria das gestantes estudadas encontravam-se consumindo carboidratos $(73,9 \%)$ e lipídeos $(52,2 \%)$ dentro dos intervalos aceitáveis de distribuição dos macronutrientes pelas calorias da dieta e que 56,5\% consumiam proteínas acima da ingestão dietética recomendada (RDA), o que sugere grande probabilidade de ingestão adequada de proteínas. No entanto, ao se analisar o consumo diário de energia total, observou-se $52,2 \%$ das gestantes ingerindo energia abaixo da necessidade mínima calculada, mostrando um déficit de ingestão energética na população.

$\mathrm{Na}$ associação entre os tercis da população para o consumo de energia e macronutrientes, oriundos de processados e ultraprocessados, às características de estado nutricional materno, os resultados significativos do Qui-Quadrado de Pearson são mostrados na Tabela 3.

Verificou-se que mulheres nos tercis superiores de consumo de carboidratos oriundos de processados e ultraprocessados possuem maior chance de atingir as necessidades energéticas recomendadas. Observou-se ainda que existe maior probabilidade de inadequação do ganho de peso gestacional para a insuficiência, quanto maior fossem os consumos de proteínas e lipídeos oriundos de processados e ultraprocessados na dieta das gestantes.

Da mesma forma, quanto maiores os percentuais que os processados e ultraprocessados representarem do consumo de energia em relação à energia total diária ingerida, maior a tendência de inadequação do ganho de peso gestacional (insuficiência ou excesso) na população de gestantes estudadas. Observou-se ainda que $65,85 \%$ das gestantes estudadas consumiam mais de $76 \%$ dos lipídeos; $69 \%$ da energia e $75 \%$ da proteína da dieta oriundos de alimentos processados e ultraprocessados. 
TABELA 3. Associações entre os tercis da população para o consumo de energia e macronutrientes, oriundos de processados e ultraprocessados, às características de estado nutricional materno em 46 gestantes acompanhadas na atenção básica do município de Gurupi -TO, 2018.

\begin{tabular}{ccccc}
\hline Grupos & \multicolumn{1}{c}{ Variáveis } & Valor $\mathbf{p}$ \\
\hline $\begin{array}{c}\text { Gramas de } \mathrm{CHO} \text { oriundos de } \\
\text { processados }+u l t r a p r o c e s s a d o s\end{array}$ & Alcance das Necessidades Energéticas & \\
& & Estimadas & & $0,025^{*}$ \\
& Insuficiente & Adequado & Excessivo & \\
$1^{\circ}$ tercil $(48,6$ a $198,8 \mathrm{~g}) \mathrm{n}=16$ & 11 & 3 & 2 & \\
$2^{\circ}$ tercil $(198,9$ a $306,5 \mathrm{~g}) \mathrm{n}=15$ & 10 & 5 & 0 \\
$3^{\circ}$ tercil $(306,6$ a $895 \mathrm{~g}) \mathrm{n}=15$ & 3 & 10 & 2 & \\
\hline
\end{tabular}

Gramas de proteínas oriundos de processados+ultraprocessados

Estado Nutricional Gestacional

$0,033^{*}$

$$
\begin{aligned}
& 1^{\circ} \text { tercil }(31,9 \text { a } 54,4 \mathrm{~g}) \mathrm{n}=14 \\
& 2^{\circ} \text { tercil }(54,5 \text { a } 88,9 \mathrm{~g}) \mathrm{n}=14 \\
& 3^{\circ} \text { tercil }(89 \text { a } 169 \mathrm{~g}) \mathrm{n}=13
\end{aligned}
$$

Percentual de PTN oriundos de processados+ultraprocessados

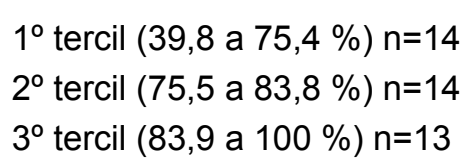

\section{Baixo Peso Eutrófico Sobrepeso Obeso}

$\begin{array}{llll}0 & 9 & 3 & 2\end{array}$

$\begin{array}{llll}6 & 4 & 4 & 0\end{array}$

$\begin{array}{llll}5 & 2 & 3 & 3\end{array}$

\section{Ganho de Peso na Gestação}

$0,003^{*}$

$\begin{array}{ccc}\text { Insuficiente } & \text { Adequado } & \text { Excessivo } \\ 4 & 8 & 2 \\ 9 & 0 & 5 \\ 10 & 2 & 1\end{array}$

Ganho de Peso na Gestação

processados+ultraprocessados

$1^{\circ}$ tercil $(49,1$ a $69,1 \%) n=14$

Adequado

7

Inadequado 7

$0,021^{*}$

$2^{\circ}$ tercil $(69,2$ a $80,5 \%) n=14$

$3^{\circ}$ tercil $(80,6$ a $90,1 \%) n=13$

\section{Percentuais de LIP oriundos de processados+ultraprocessados}

$$
\begin{aligned}
& 1^{\circ} \text { tercil }(31,1 \text { a } 76,7 \%) n=14 \\
& 2^{\circ} \text { tercil }(76,8 \text { a } 87,9 \%) n=14 \\
& 3^{\circ} \text { tercil }(88 \text { a } 98,4 \%) n=13
\end{aligned}
$$

2

1

12

12

\section{Ganho de Peso na Gestação}

Insuficiente

3
12
8

3

8

Adequado
7
1
2

Excessivo

$\begin{array}{ll}4 & 0,011^{*} \\ 1 & \\ 3 & \end{array}$

Fonte: Dados da Pesquisa.

Nota: * Significativo pelo teste do Qui-quadrado de Pearson a 5\%.

\section{DISCUSSÃO}

Na população de gestantes estudadas observou-se alta prevalência de ganho de peso gestacional insuficiente, que esteve diretamente associado ao elevado consumo de proteínas e lipídeos oriundos de alimentos ultraprocessados. Sabe-se que o ganho inadequado de peso na gestação é fator de risco para desfechos negativos da gestação, como a prematuridade e o baixo peso ao nascer 
(FERNANDES et al., 2019). Além disso, o consumo de processados e ultraprocessados impacta negativamente 0 teor de micronutrientes da dieta, tornando-a deficiente no fornecimento de, principalmente, ferro, zinco e vitamina $A$, nutrientes cujas carências estão entre os problemas nutricionais que mais afetam crianças e gestantes, ocasionando retardo do crescimento e do desenvolvimento infantil e aumento da mortalidade fetal e materna (LOUZADA et al., 2015).

A literatura sugere que ganho de peso na gestação está diretamente associado ao consumo de nutrientes e padrões alimentares inadequados durante a gestação podem trazer efeitos adversos à saúde do binômio, tais como sintomas depressivos na gestante, depressão pós-parto, desenvolvimento de pré-eclâmpsia grave, aborto espontâneo recorrente, diabetes gestacional, baixo peso ao nascer, nascimento de bebês pequenos para a idade gestacional e presença de defeitos congênitos (WILLIAMS et al., 2016; ZHAO et al., 2017; YANG et al., 2017; LI et al., 2017; MARKHUS et al., 2018).

Outra constatação do presente estudo, foi que o estado nutricional das gestantes tendeu ao declínio da eutrofia para o baixo peso, quando se comparam os períodos pré-gestacional e a da gestação atual. A literatura destaca que o estado nutricional materno inadequado pode ser causa direta de baixo peso ao nascer e restrição de crescimento intrauterino (MARTINS; BENICIO, 2011).

Por outro lado, o excesso de peso também esteve prevalente em 41,3\% das gestações e este pode predispor à retenção de peso no pós-parto, incrementando os índices de sobrepeso e obesidade entre mulheres em idade fértil (GOMES et al., 2014).

Os resultados do presente estudo foram semelhantes aos de estudos realizados com gestantes, na atenção primária de saúde, de outros municípios do Brasil, para baixo peso (ROSA, et al., 2014; MEIRELES et al., 2016) e excesso de peso (PROCTER; CAMPBELL, 2014; MEIRELES et al., 2016).

No presente estudo pode-se observar também que os percentuais expressivos de ingestão de produtos alimentícios processados e ultraprocessados afetaram, diretamente, tanto a relação entre os macronutrientes e a quantidade de energia diária ingerida total, como o estado nutricional materno. Tais resultados, confirmam os prejuízos à saúde humana atribuídos ao consumo predominante de processados e ultraprocessados na dieta (MARTINS et al., 2013; CRIVELLENTI et al., 2017), e endossam o impacto negativo no estado nutricional durante a gestação.

Os alimentos processados em geral, sobretudo os ultraprocessados apresentam uma tendência de maior participação na composição da dieta atual das populações. Esses alimentos, além de alta densidade energética, apresentam ainda excesso de adição de açúcares, gorduras totais e saturadas, estando relacionados ao surgimento de doenças crônicas não transmissíveis como o diabetes, a hipertensão, as doenças cardiovasculares e o câncer (LOUZADA et al., 2015; ZOBEL et al., 2016; MARTINS; FARIA, 2018) .

De acordo com o Guia Alimentar da População Brasileira, os alimentos que devem compor a base da alimentação da população são os alimentos in natura ou minimamente processados, visto que contribuem para melhor promoção da saúde, contendo maiores teores de fibras, vitaminas e minerais. Já o consumo de alimentos processados e ultraprocessados é desencorajado, pois promovem um desbalanço nutricional e podem gerar uma cascata de distúrbios metabólicos e fisiológicos (WHO, 2013). As recomendações oficiais priorizam a redução do consumo de alimentos ultraprocessados, bem como o aumento das práticas culinárias e da ingestão dos alimentos in natura e minimamente processados (WHO, 2013). 
Como limitação desse estudo destacou-se não terem sido investigadas as associações do consumo alimentar com variáveis socioeconômicas, por exemplo, de renda, escolaridade e ocupação, uma vez que o consumo de alimentos industrializados também está relacionado a fatores como custo, durabilidade, facilidade de armazenamento, falta de informação sobre o risco à saúde, facilidade de preparo e consumo; tendo sido evidenciado na literatura um maior consumo de ultraprocessados por famílias de renda mais baixa (ESTIMA et al., 2011).

Observou-se que, apesar das gestantes do presente estudo obterem ganho de peso insuficiente, o consumo de processados e ultraprocessados contribuiu para o alcance das necessidades energéticas estimadas. Isso deve-se ao fato de que esses produtos alimentícios são na totalidade constituídos de "calorias vazias", ou seja, sem quaisquer resquícios de nutrientes na composição, não fornecendo nenhum valor nutritivo.

Diante do exposto, desencorajar o consumo de alimentos ultraprocessados é, indubitavelmente, uma questão de saúde pública, uma vez que resgata os alimentos naturais e regionais como também a qualidade de vida da população.

\section{CONCLUSÃO}

O consumo dos produtos alimentícios processados e ultraprocessados contribuiu para o alcance das necessidades energéticas estimadas na população de gestantes de Gurupi, uma vez que apresentam alta densidade energética na composição. No entanto, o consumo de macronutrientes advindos desses tipos de alimentos correlacionaram-se, em sua maioria, de forma negativa ao estado nutricional materno, sendo o ganho de peso e o índice de massa corporal afetados significativamente.

A avaliação do consumo alimentar, levando-se em consideração o grau de processamento dos alimentos consumidos durante a gestação é fundamental para o estabelecimento de estratégias para melhora do perfil de consumo de alimentos nesse grupo populacional, por meio do empoderamento das gestantes, afim de se desestimular o consumo de alimentos processados e ultraprocessados e resgatar os alimentos regionais in natura e as práticas culinárias da cultura local.

\section{REFERÊNCIAS}

ATAlAh, E. S.; CASTILlo, C. L.; RENÉ, C. S.; AldeA, A. P. Propuesta de un nuevo estándar de evaluación nutricional em embarazadas. Revista Medica de Chile, v. 125, n. 12, p. 1429-1436, 1997. Disponível em:<https://biblat.unam.mx/pt/revista/revista-medica-de-chile/articulo/propuesta-deun-nuevo-estandar-de-evaluacion-nutricional-en-embarazadas>

BRASIL. Ministério do Planejamento, Orçamento e Gestão. Instituto Brasileiro de Geografia e Estatística. Diretoria de Pesquisas. Coordenação de Trabalho e Rendimento. Pesquisa de orçamentos familiares 2008-2009 - Tabelas de Composição Nutricional dos Alimentos Consumidos no Brasil. Rio de Janeiro: IBGE; 2011. Disponível em: <https://biblioteca.ibge.gov.br/visualizacao/livros/liv50002.pdf>

CAMPOS, C. A. S.; MALTA, M. B.; NEVES, P. A. R.; LOURENÇO, B. H.; CASTRO, M. C. et al. Ganho de peso gestacional, estado nutricional e pressão arterial de $\mathrm{g}$ estantes. Revista de Saúde Pública, v. 53, 2019. Disponível em: <https://www.scielosp.org/article/rsp/2019.v53/57/pt/\#> doi: https://doi.org/10.11606/ S1518-8787.2019053000880. 
COMINETTI, C.; COZZOLINO, S. M. F. Recomendações de Nutrientes. São Paulo: International Life Sciences Institute do Brasil, 2017. Disponível em: https://ilsibrasil.org/wp-content/uploads/sites/9/2017/07/FascículoRECOMENDACOES-DE-NUTRIENTES.pdf

CRISPIM, S. P.; FISBERG, R. M.; ALMEIDA, C. C. B.; NICOLAS, G.; KNAZE, V. et al. Manual fotográfico de quantificação alimentar. 1ed. Curitiba: UFPR, 2017. 147p. Disponível em:<http://www.ucv.edu.br/biblioteca/livro-virtual/manualfotografico-quantificacao-alimentar.pdf>

CRIVELLENTI, L. C; ZUCCOLOTTO, D. C. C; SATORELLI, D. S. Desenvolvimento de um Índice de Qualidade da Dieta Adaptado para Gestantes. Revista de Saúde Pública, v. 52, n. 59, 2017. Disponível em: <http://www.scielo.br/scielo.php? pid $=S 003489102018000100249 \&$ script=sci_arttext\&tlng=pt $>$. doi:10.11606/s15188787.2018052000184

CUNHA, L. R.; PRETTO, A. D. B.; BAMPI, S. R.; SILVA, J. M. G. C.; MOREIRA, A. M. Avaliação do estado nutricional e do ganho de peso de gestantes atendidas em uma unidade básica de saúde de Pelotas-RS. Revista Brasileira de Obesidade, Nutrição e Emagrecimento, v. 10, n. 57, p. 123-132, 2016. Disponível em: < http:// www.rbone.com.br/index.php/rbone/article/view/424/384>

ESTIMA, C. C. P.; PHILIPPI, S. T.; ARAKI, E. L.; LEAL, G. V. S.; MARTINEZ, M. F.; ALVARENGA, M. S. Consumo de bebidas e refrigerantes por adolescentes de uma escola pública. Revista Paulista de Pediatria, v. 29, n. 1, p. 41-5, 2011.

FERNANDES, D. C.; CARRENO, I.; SILVA, A. A.; GUERRA, T. B.; ADAMI, F. S. Relação entre o estado nutricional pré-gestacional e o tipo de processamento de alimentos consumidos por gestantes de alto risco. Revista Brasileira Saúde Materno Infantil, v. 19, n. 2, 2019. Disponível em: <http://www.scielo.br/scielo.php? pid=S151938292019000200351\&script=sci_arttext\&tlng=pt $>$. doi:10.1590/180693042019000200006.

GOMES, R. N. S.; GOMES, V. T. S.; CALDAS, D. R. C.; LAGO, E. C.; CAMPOS, F. K. L. et al. Avaliação do estado nutricional de gestantes atendidas em unidades básicas de saúde de Caxias/Ma. Revista Interdisciplinar, v. 7, n. 4, p. 81-90, 2014.

LI, N.; WU, H. M.; HANG, F.; ZHANG, Y. S.; LI, M. J. et al. Women with recurrent spontaneous abortion have decreased $25(\mathrm{OH})$ vitamin $\mathrm{D}$ and VDR at the fetalmaternal interface. Brazilian Journal of Medical and Biological Research, v. 50, n. 11, 2017 Disponível em: <http://www.scielo.br/pdf/bjmbr/v50n11/1414-431X-bjmbr1414-431X20176527.pdf>. doi: 10.1590/1414-431X20176527

LOUZADA, M. L. C.; MARTINS, A. P. B; CANELA, D. S.; BARALDI, L. G.; LEVY, R. $B$. et al. Alimentos ultraprocessados e perfil nutricional da dieta no Brasil. Revista de Saúde Pública, v. 40, n. 38, p.1-11, 2015. Disponível em: http://www.scielo.br/pdf/rsp/v49/pt_0034-8910-rsp-S0034-89102015049006132.pdf

MARKHUS, M. W.; DAHL, L.; MOE, V.; ABEL, M. H.; BRANTSAETER, A. L. et al. Maternal lodine Status is Associated with Offspring Language Skills in Infancy and 
Toddlerhood. Nutrients, v. 10, n. 9, 2018. Disponivel em: <https://www.ncbi.nlm.nih.gov/pubmed/30205599>. doi: 10.3390/nu10091270.

MARTINS, A. P. B.; BENICIO, M. H. D. Influência do consumo alimentar na gestação sobre a retenção de peso pós-parto. Revista de Saúde Pública, v. 45, n. 5, p. 87077, 2011. Disponivel em: <http://www.scielo.br/pdf/rsp/v45n5/2493.pdf $\geq$

MARTINS, A. P. B; LEVY, R. B.; CLARO, R. F; MOUBARAC, J. C; MONTERIO, C. A. Participação crescente de produtos ultraprocessados na dieta brasileira (19872009). Revista de Saúde Pública, v. 47, n.4, p. 656-65, 2013. Disponível em: $<$ http://www.scielo.br/scielo.php?pid=S0034-

89102013000400656\&script=sci_abstract\&tlng=pt> doi: $\quad$ 10.1590/S00348910.2013047004968

MARTINS, P. F. A; FARIA, L. R. C. Alimentos ultraprocessados: uma questão de saúde pública. Comunicação em Ciências da Saúde, v. 29, n. 1, p. 14-17, 2018. Disponivel

em: <http://bvsms.saude.gov.br/bvs/periodicos/ccs_artigos/v29_supl_alimentos_ultraproc essados.pdf>

MEIRELES, J. F. F.; NEVES, C. M.; DE CARVALHO P. H. B.; FERREIRA, M. E. C. Satisfação corporal, idade gestacional e estado nutricional em gestantes. ABCS Health Sciences, v. 41, n. 1, p. 23-28, 2016.

MONTEIRO, C. A.; CANNON, G.; LEVY, R.; MOUBARAC, J. C.; JAIME, P. et al. Classificação dos alimentos. Saúde Pública: NOVA. A estrela brilha. World Nutrition, v. 7, p. 28-40, 2016. Disponível em:<http://www.cookie.com.br/site/wpcontent/uploads/2016/12/NOVA-Classificação-dos-Alimentos.pdf>.

NÚCLEO DE ESTUDOS E PESQUISAS EM ALIMENTAÇÃO. Universidade Estadual de Campinas. Tabela Brasileira de Composição de Alimentos - TACO. Campinas: Unicamp/NEPA, 2011.2 Disponível em: https://www.cfn.org.br/wpcontent/uploads/2017/03/taco_4_edicao_ampliada_e_revis ada.pdf

PHILIPPI, S. T. Tabela de Composição de Alimentos: Suporte para Decisão Nutricional. 4 ed. São Paulo: Metha; 2015. 164 p.

PROCTER, S. B.; CAMPBELLCG. Position of the Academy of Nutrition and Dietetics: nutrition and lifestyle for a healthy pregnancy outcome. The Journal of the Academy of Nutrition and Dietetics, v. 114, n. 7, p. 1099-103, 2014. Disponível em: <https://www.ncbi.nlm.nih.gov/pubmed/24956993>. doi: 10.1016/j.jand.2014.05.005.

RASMUSSEN, K. M.; YAKTINE, A. L. Institute of Medicine. Weight Gain During Pregnancy: Reexamining the Guidelines. Washington, DC: The National Academies $\quad$ Press, 2009.2 Disponível em:<https://www.cbsnews.com/htdocs/pdf/052809_pregnancy.pdf>

ROSA, R. L.; MOLZ, P.; PEREIRA, C. S. Perfil nutricional de gestantes atendidas em uma unidade básica de saúde. Cinergis, v. 15, n. 2, p.98-102, 2014. 
WILLIAMS, J. A.; ROMERO, V. C.; CLINTON, C. M.; VAZQUEZ, D. M.; MARCUS, S. $M$. et al. Vitamin $D$ levels and perinatal depressive symptoms in women at risk: a secondary analysis of the mothers, omega-3, and mental health study. BMC

Pregnancy and Childbirth, v. 16, 2016. Disponível em: <https://www.ncbi.nlm.nih.gov/pmc/articles/PMC4971719/>. doi: 10.1186/s12884016-0988-7

WORLD HEALTH ORGANIZATION. Department of Chronic diseases and Health Promotion. Global strategy on diet, physical activity and health. Geneva: WHO, 2013.

YANG, J.; CHENG, Y.; PEI, L.; JIANG, Y.; LEI, F. et al. Maternal iron intake during pregnancy and birth outcomes: a cross-sectional study in Northwest China. British Journal of Nutrition, v. 117, n. 6, p. 862-871, 2017. Disponível em: <https://www.ncbi.nlm.nih.gov/pubmed/28393737>. doi: $10.1017 /$ S0007114517000691

ZHAO X.; FANG, R.; YU, R.; CHEN, D.; ZHAO, J. et al. Maternal Vitamin D Status in the Late Second Trimester and the Risk of Severe Preeclampsia in Southeastern China. Nutrients, v. 9, n. 2, p. 138, 2017. Disponível em: < https://www.ncbi.nlm.nih.gov/pmc/articles/PMC5331569/>. doi: 10.3390/nu9020138

ZOBEL, E. H; HANSEN, T. W; ROSSING, P.; VON SCHOLTEN, B. J. Global Changes in Food Supply and the Obesity Epidemic. Current Obesity Reports, v. 5, n. 4, p. 449-455, 2016. 